\title{
Posterior Scleritis: About A Case
}

\author{
J. B. Salem, M. Khammaily, S. Dahi, B. Houda, Y. Mouzari, K. Reda, and A. Oubaaz
}

\begin{abstract}
Posterior scleritis is a rare and often unknown condition that can simulate many conditions including intraocular tumor or orbital pathology. We report the case of a 44-year-old patient with left eye pain and redness followed by a significant decrease in visual acuity. Clinical examination showed papillary hyperemia, chorioretinal folds at the posterior pole, venous tortuosity, and absence of hyalitis or vasculitis in the fundus oculi associated angiography retinal. Orbital-cerebral MRI and B ultrasound allowed the diagnosis of posterior scleritis.
\end{abstract}

Index Terms - Posterior scleritis, MRI orbitofrontal brain, corticosteroid.

\section{INTRODUCTION}

Posterior scleritis is an infectious or inflammatory scleral disease, located behind the equator. The ophthalmological manifestations are very polymorphic and not very specific, which leads to a diagnostic difficulty [1].

\section{PATIENT AND OBSERVATION}

Patient aged 44, hospitalized in the internal medicine department for exploration of a deterioration of the general state, vomiting, and ocular redness with the following history: deficiency anemia and dysphagia evolving for 2 years. The history of the disease goes back to a week before its hospitalization, marked by insomnia headaches resistant to the analgesic treatments, associated with vomiting and ocular signs associating with a decrease in visual acuity, bilateral visual blurs (more accentuated on the side right), eye pain and redness. The patient was initially hospitalized in the neurology department where a brain CT scan was

Published on June 10, 2020

J. B. Salem, Mohammed V Military Instructional Hospital, Faculty of Medicine and Pharmacy Mohammed V University, Morocco.

(corresponding e-mail: joumanybss ${ }^{@}$ gmail.com).

M. Khammaily, Mohammed Military Instruction Hospital V, Faculty of Medicine and Pharmacy Mohammed V University, Morocco.

(e-mail: dahisidi2014@gmail.com).

S. Dahi, Mohammed V Military Instruction Hospital, Faculty of Medicine and Pharmacy Mohammed V University, Morocco.

(e-mail: dahisidi2014@gmail.com).

B. Houda: Mohammed V Military Instructional Hospital, Faculty of Medicine and Pharmacy Mohammed V University, Morocco.

Y. Mouzari, Department of Ophthalmology, Military Hospital of Instruction Mohammed V, Faculty of Medicine and Pharmacy Mohammed V University, Morocco.

(e-mail: ys.mouzari@gmail.com).

K. Red, Mohammed V Military Instruction Hospital, Faculty of Medicine and Pharmacy Mohammed V University, Morocco,

(e-mail: mkarimreda ${ }^{\circledR}$ gmail.com).

A. Oubaaz, Ophthalmology Service, Military Instruction Hospital Mohammed V, Faculty of Medicine and Pharmacy Mohammed V University, Morocco.

(e-mail: aoubaaz@gmail.com). done in an emergency but returned to normal.

The ophthalmic examination revealed visual acuity: with the right eye (OD) of 4/10, with the left eye (OG) of $3 / 10$, the absence of signs of uveitis or anterior scleritis, an eye tone at $16 \mathrm{mmHg}$ ODG and the back of the eye (Fig. 1) papillary hyperemia with venous pulse present, chorioretinal folds at the posterior pole (PP), venous tortuosity and an absence of hyalitis or vasculitis.

The biological assessment is in favor of an artery-forming microcytic hypochromic anemia. The infectious investigation is negative (QuantiFERON, syphilis serology, hepatitis serology, Herpes, Herpes, HIV, EBV, brucellosis serology, atypical germ serology) and the immunological investigation (AAN; ANCA; ACL; Cryoglobulin) is also negative. Additional exams, namely ultrasound B (Fig. 2), are in favor of bilateral posterior scleritis with posterior thickening of the 2 eyeballs. Retinal angiography (Fig. 3) finds venous tortuosities, choroidal folds, and a retinal serous detachment (DSR). OCT (Fig. 4) showed the presence of a retinal serous detachment (DSR). Orbital MRI (Fig. 5) showed a thickened and regular appearance of the posterior segment wall, continuing with a thickening of the proximal part of the optic nerve for both the right and left eyeball compatible with bilateral posterior scleritis. The diagnosis of idiopathic bilateral posterior scleritis was accepted, and the patient subsequently received a bolus of corticosteroids, followed by an oral relay in decreasing doses. The evolution was quickly favorable with a rapid improvement in the symptomatology and visual acuity which rose to 6/10 in far vision (VL) on the Snellen scale and in Parinaud 3 in meadows vision (VP). The control ultrasound showed a sclera of normal thickness.

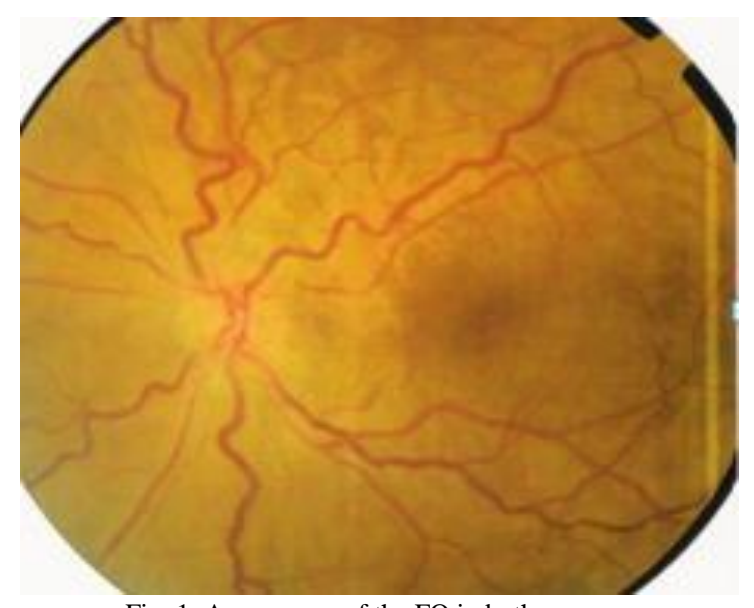

Fig. 1. Appearance of the FO in both eyes. 


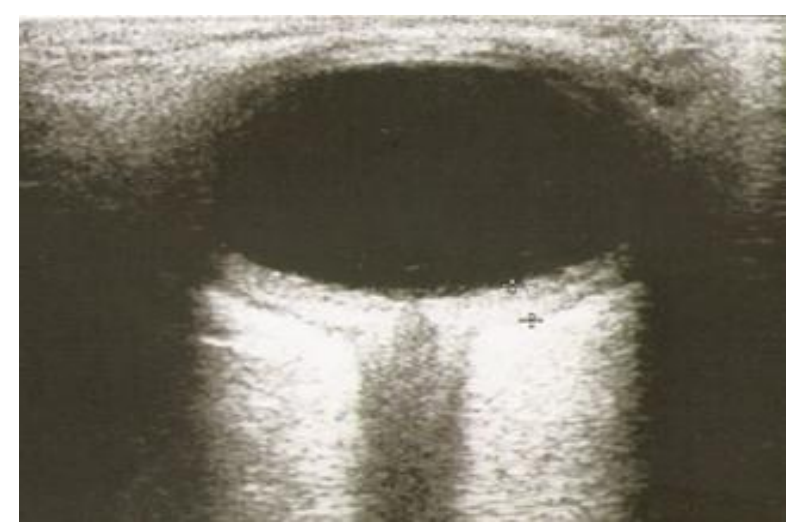

Fig. 2. Ultrasound image: posterior scleritis post thickening of the 2 globes.

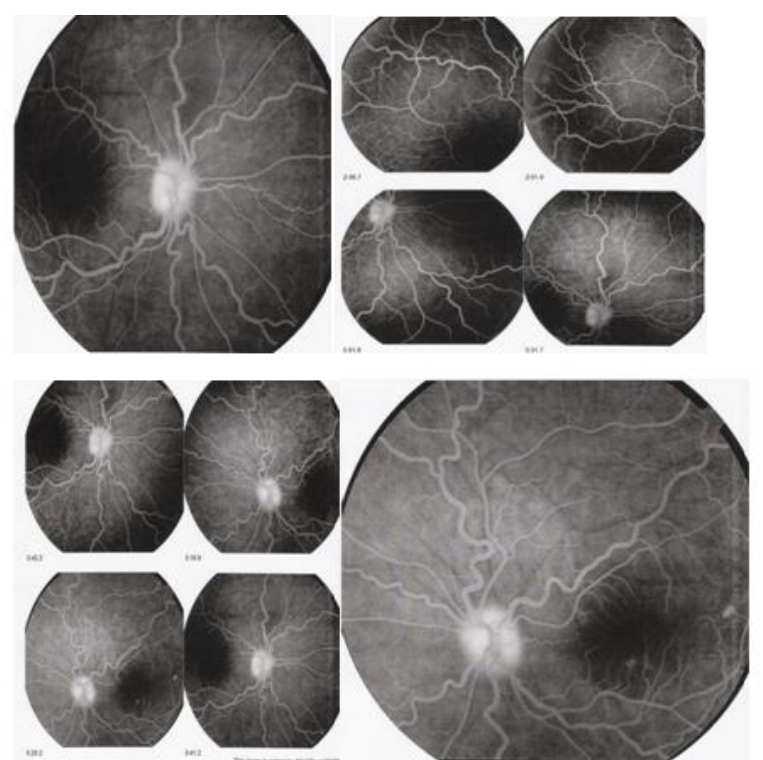

Fig. 3. Fluorescein angiography Finds venous tortuosities, choroidal folds and DSR.

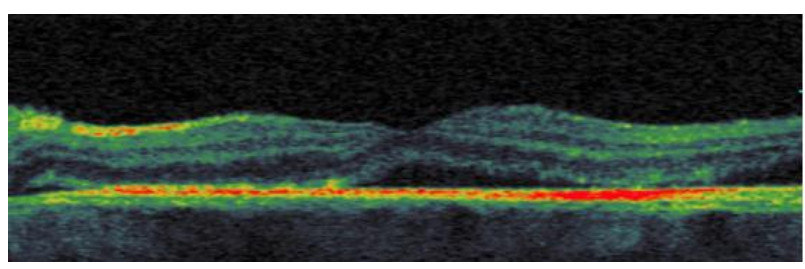

Fig. 4. OCT: serous retinal detachment aspect.
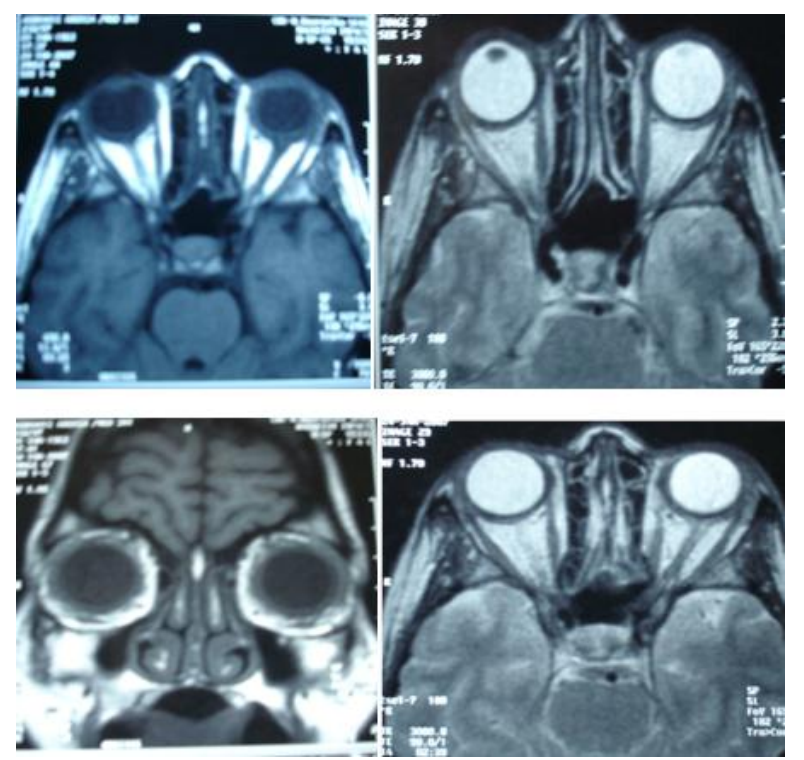

Fig. 5. MRI: thickening of the wall of the post segment and the proximal part of the optic nerve.

\section{DISCUSSION}

Scleritis is a rare disease, with an estimated prevalence of 6 cases per 100,000 in the general population [1]. Posterior scleritis is an infectious or inflammatory scleral disease, located behind the equator. It is less common than anterior scleritis (2 to $12 \%)$; relatively rare, however, its frequency would be underestimated due to the difficulty of its diagnosis linked to the inaccessibility of the sclera posterior to direct examination [1], [2]. The involvement is unilateral in 67 to $90 \%$ of cases with a female predominance according to the data in the literature [2], [3]. The ophthalmological manifestations are very polymorphic and not very specific: drop-in vision with a red and painful eye accompanied or no palpebral edema, spontaneous retrobulbar and periorbital pain, accentuated in eye movements, with irradiation towards the cheek, diplopia, and ptosis can be observed in severe cases [4], [5]. The association of anterior scleritis with posterior scleritis was observed in $70 \%$ of cases in the literature. The evolution can sometimes be marked by serious intraocular complications: choroiditis with pseudo-tumor-like appearance, hyalitis, and exudative retinal detachment. Several lesions can be indicative of posterior scleritis: chorioretinal and retinal folds, RSD, subretinal masses, choroidal uplift, papillary edema, cystoid macular edema, ciliocorchoid detachments [6]. Association with posterior, intermediate uveitis or retinal vasculitis is possible. Mode B ultrasound: is a simple and essential examination for the diagnosis of posterior scleritis. It visualizes the presence of a hyperechogenic scleral thickening with hyperreflectivity, retrobulbar edema, and especially a pathognomonic sign (the T-shaped image) by the horizontal hypoechogenicity of the sub-tenonian edema and by that vertical of the optic nerve [7]. Fluorescein angiography: allows us to visualize a DSR: delay of choroidal fluorescence at early times, vanishing points (pinpoints) at average times with a heterogeneous filling of DSR at late times. Other signs can also be seen: chorioretinal folds (alternating hyper and hyperfluorescent streaks), subretinal mass, papillary edema, and also cystoid macular edema [7], [8].

Indocyanine green angiography (ICG) makes it possible to assess choroidal participation by objectifying a delay in choroidal perfusion, areas of diffuse choroidal hyperfluorescence in the 2 intermediate and late stages and dilation of the choroidal veins [9]. The signs observed on indocyanine green angiography are neither pathognomonic nor specific for posterior scleritis [2]. MRI and CT are useful for confirming the diagnosis in doubtful cases and differentiating an inflammatory disease from an infiltrative process. It shows scleral thickening with significant enhancement after injection of the contrast agent and inflammation of the oculomotor muscles and / or other periocular tissues. The differential diagnosis of posterior scleritis arises with many affections such as Vogt-KoyanagiHarada syndrome, Malignant melanoma of the choroid, Choroidal metastases, etc. [2]. The clinical context, fluorescein angiography, B-mode oculo-orbital ultrasound, CT, and MRI are often used to decide. The etiologies of posterior scleritis [4], [5], [7] are system diseases: rheumatic affections, Rheumatoid arthritis, SPA, Still's a disease, Psoriasis; connectivities: Lupus, Scleroderma, Chronic 
atrophic polychondritis; chronic inflammatory enteropathies: Crohn, $\mathrm{RCH}$; vasculitis: Wegener's granulomatosis, knotty periarteritis; Behcet, infections: viral: Herpes simplex, Shingles, Mumps; bacterial: Tuberculosis, Syphilis, Leprosy, Brucellosis, Lyme disease, and Idiopathic in $56 \%$ of cases. The visual prognosis of posterior scleritis depends on several factors including the duration of progression and the severity of the disease, the extension of the inflammation to the optic nerve, macular complications, the existence of an associated anterior involvement, presence of an underlying systemic disease and the existence of complications such as cataracts or glaucoma [10].

The treatment involves several medications and has 2 components: fight against inflammation and treat the causative pathology [9] - [12].

There are various means: non-steroidal anti-inflammatory drugs (NSAIDs): 50mg X3 / d for 15 days then reduction by half for 1 to 2 weeks after clinical recovery, Corticotherapy: if resistance to NSAIDs or recurrences or association with a systemic disease; can be administered locally, orally or intravenously, Immunosuppres.

\section{CONCLUSION}

Posterior scleritis is often difficult to diagnose, it must be brought up in the presence of any inflammatory and painful eye symptomatology which does not prove its origin. Signs of borrowing are common. Ultrasound B and MRI are the examination of choice for the diagnosis of this oftenoverlooked condition. Response to anti-inflammatory therapy is generally excellent.

\section{REFERENCES}

[1] Artifoni M, Rothschild P-R, Brézin A, Guillevin L, Puéchal X. Ocular inflammatory diseases associated with rheumatoid arthritis. Nat Rev Rheumatol. 2014;10(2):108-116.

[2] Daniel Diaz J, Sobol EK, Gritz DC. Treatment and management of scleral disorders. Surv Ophthalmol. 2016;61(6):702-717.

[3] Honik G, Wong IG, Gritz DC. Incidence and prevalence of episcleritis and scleritis in Northern California. Cornea. 2013;32(12):1562-1566.

[4] Sims J. Scleritis: presentations, disease associations and management Postgrad Med J. 2012;88(1046):713-718.

[5] Oray M, Meese H, Foster CS. Diagnosis and management of noninfectious immune-mediated scleritis: current status and future prospects. Expert Rev Clin Immunol. 2016;12(8):827-837.

[6] Wieringa WG, Wieringa JE, ten Dam-van Loon NH, Los LI. Visual outcome, treatment results, and prognostic factors in patients with scleritis. Ophthalmology. 2013;120(2):379-386.

[7] Gonzalez-Gonzalez LA, Molina-Prat N, Doctor P, Tauber J, Sainz de la Maza M, Foster CS. Clinical features and presentation of posterior scleritis: a report of 31 cases. Ocul Immunol Inflamm. 2014;22(3):203-207.

[8] Wald KJ, Spaide R, Patalano VJ, Sugin S, Yannuzzi LA. Posterior Scleritis in Children. American Journal of Ophthalmology. 1992;113(3):281-286.

[9] Perri P, Mazzeo V, De Palma P, Pastena B, Police G, Ravalli L, et al. Posterior scleritis: ultrasound findings in two cases. Ophthalmologica. 1998;212 Suppl 1:110-112.

[10] Posterior Scleritis: Analysis of Epidemiology, Clinical Factors, and Risk of Recurrence in a Cohort of 114 Patients.

[11] Stem MS, Todorich B, Faia LJ. Ocular Pharmacology for Scleritis: Review of Treatment and a Practical Perspective. J Ocul Pharmacol Ther. 2017;33(4):240-246.

[12] Nascimento H, França M, García LG, Muccioli C, Belfort R. Subconjunctival dexamethasone implant for non-necrotizing scleritis. J Ophthalmic Inflamm Infect. 2013;3(1):7. 